EESTI NSV TEADUSTE AKADEEMIA TOIMETISED, 26. KOIDE FUOSIKA * MATEMAATIKA, 1977, NR. 4

ИЗВЕСТИЯ АКАДЕМИИ НАУК ЭСТОНСКОИ ССР. ТОМ 26 ФИЗИКА * МАТЕМАТИКА. 1977, № 4

B. ВАНАTOA, М. ЛАATC,

A. КАРТУШИНСКИИ, Ф. ФРИШМАН

\title{
ИНТЕГРАЛЬНЫИ МЕТОД РАСЧЕТА ДВУХФАЗНОЙ НЕРАВНОВЕСНОЙ СТРУИ
}

В извеетной работе С. Патанкара и Д. Сполдинга [ $\left.{ }^{1}\right]$ теории однофазного пограничного слоя делятся на полные и явно интегральные. Характеристиками первых являются полуэмпирические гипотезы-для турбулентной вязкости и численные методы решений дифференциальных уравнений в частных производных переноса различных субстанций, а характеристиками вторых - эмпирические соотношения, связывающие интегральные параметры слоя, и численные методы решения обыкновенных дифференциальных уравнений, описывающих интегральные свойства пограничного слоя. Конечно, полные теории предпочтительнее, однако в тех случаях, когда численное решение уравнений. в частных производных затруднительно, в явно интегральных методах можно использовать соотношения, содержащие турбулентные касательные напряжения и такие же полуэмпирические гипотезы, как и в полных теориях. Это делает интегральные методы более обоснованными.

Интегральные соотношения получаются путем интегрирования исходных дифференциальных уравнений

$$
\begin{aligned}
& \frac{\partial}{\partial x} y^{\varepsilon} \mathrm{Q} \alpha U_{s}^{2}+\frac{\partial}{\partial x} y^{\varepsilon} \mathrm{Q} U^{2}+\frac{\partial}{\partial y} y^{\varepsilon} \mathrm{Q} \alpha U_{s} V_{s}+\frac{\partial}{\partial y} y^{\varepsilon} \mathrm{Q} U V= \\
& =-\frac{\partial}{\partial y} y^{\varepsilon} \mathrm{Q} u^{\prime} v^{\prime}-\frac{\partial}{\partial y} y^{\varepsilon} \mathrm{Q} \alpha u_{s}^{\prime} v_{s}^{\prime}-\frac{\partial}{\partial y} y^{\varepsilon} \mathrm{Q}^{\prime} v^{\prime}{ }_{s} U_{s},
\end{aligned}
$$

$\frac{\partial}{\partial x} y^{\varepsilon} \mathrm{Q} \alpha U_{s}^{2}+\frac{\partial}{\partial y} y^{\varepsilon} \mathrm{Q}^{\prime} U_{s} V_{s}=-\frac{\partial}{\partial y} y^{\varepsilon} \mathrm{Q} \alpha u_{s}^{\prime} v_{s}^{\prime}-\frac{\partial}{\partial y} y^{\varepsilon} \alpha^{\prime} v_{s}^{\prime} U_{s} \pm \frac{\varrho \alpha}{m_{p}} f$,

$$
\frac{\partial}{\partial x} y^{\varepsilon} \varrho \alpha U_{s}+\frac{\partial}{\partial y} y^{\varepsilon} \varrho \alpha V_{s}=-\frac{\partial}{\partial y} y^{\varepsilon} \varrho \alpha^{\prime} v_{s}^{\prime} .
$$

Здесь $U, U_{s}, V, V_{s}$ - продольные и поперечные составляющие скоростей газа и примеси соответственно, $\varrho-$ плотность несущей фазы, $\boldsymbol{\alpha}-$ массовая концентрация примеси, $\varepsilon=1$ для осесимметричного течения, $\varepsilon=0$ для плоскопараллельного течения, $m_{p}-$ масса частицы.

После интегрирования имеем

$$
\frac{d}{d x} \int_{0}^{\infty} \varrho \alpha U_{s}^{2} y^{\varepsilon} d y+\frac{d}{d x} \int_{0}^{\infty} \mathrm{Q} U^{2} y^{\varepsilon} d y=0,
$$




$$
\begin{aligned}
& \frac{d}{d x} \int_{0}^{\infty} \varrho \alpha U_{s}^{2} y^{\varepsilon} d y= \pm \int_{0}^{\infty} \frac{\varrho \alpha}{m_{p}} f d y, \\
& \frac{d}{d x} \int_{0}^{\infty} \varrho \alpha U_{s} y^{\varepsilon} d y=0 .
\end{aligned}
$$

Здесь $f$ - сила сопротивления движению частицы.

Для определения $f$ удобно использовать данные $\left[{ }^{2}\right]$

$$
\begin{aligned}
& f=\frac{24}{\operatorname{Re}} L \frac{\pi \delta^{2}}{4} \frac{\varrho\left(U-U_{s}\right)^{2}}{2}, \\
& L=1+0,15 \operatorname{Re}^{0,687},
\end{aligned}
$$

где $\delta$ - диаметр частицы.

Соотношения, содержащие турбулентную вязкость, обычно получаются путем умножения уравнений (1) на скорость (соотношения Голубева) или радиус (соотношения Лойцянского) в степени 1, 2, 3 и последующего интегрирования. Система уравнений Голубева использована в $\left.\Gamma^{3}\right\rceil$ для расчета двухфазных равновесных турбулентных течений и в [ $\left.{ }^{4}\right]$ для расчета неравновесных течений. Уравнения в [ $\left.{ }^{4}\right]$ отличаются не только сложностью. Они содержат в правой части под интегралом неавтомодельные члены, что исключает возможность получения аналитическото решения и сушественно усложняет численное решение.

Система интегральных соотношений выглядит гораздо проще, если уравнения типа (2) использовать до какой-либо характерной линии струи. Если эта линия не граница, в уравнения войдут и турбулентные касательные напряжения. Такие методы использовались для расчета однородных струйных течений [5]. Однако существенных преимушеств не давали, так как для таких течений соотношения Голубева и Лойцянского тоже достаточно просты.

Рассмотрим лля примера основной участок осесимметричной турбулентной струи. Уравнение, содержащее турбулентные касательные напряжения, получается при интегрировании уравнения (1) до линии, на которой $\alpha=0,5 \alpha_{m}$ (индекс $m$ обозначает осевые значения)

$$
\begin{aligned}
& \frac{d}{d x} \int_{0}^{r_{\alpha}} \varrho U^{2} y^{\varepsilon} d y+\frac{d}{d x} \int_{0}^{r_{\alpha}} \varrho \alpha U_{s}^{2} y^{\varepsilon} d y-U_{0,5} \frac{d}{d x} \int_{0}^{r_{\alpha}} \varrho U y^{\varepsilon} d y- \\
& -U_{s 0,5} \frac{d}{d x} \int_{0}^{r_{\alpha}} \varrho \alpha U_{s} y^{\varepsilon} d y=\left.k_{Q} U_{m} r_{u} r_{\alpha} \frac{1+0,5 n^{2} \alpha_{m}}{1+0,5 \alpha_{m}} \frac{d U}{d y}\right|_{r_{\alpha} .}
\end{aligned}
$$

Здесь $r_{u}, r_{\alpha}$ - значения радиусов, где скорость и концентрация равны половине осевых значений; коэффициент турбулентной вязкости принят, согласно теории Прандтля, равным $k U_{m} r_{u}$, а влияние примеси на турбулентность определено по теории Абрамовича $\left[{ }^{6}\right] ; n-$ параметр, характеризующий относительную скорость частиц в турбулентном моле.

Приведем уравнение (4) к безразмерному виду для $\varepsilon=1$ :

$$
\frac{d}{d x} \varrho U_{m}^{2} r_{u}^{2} \int_{0}^{r_{\alpha} / r}\left(\frac{U}{U_{m}}\right)^{2} \frac{y}{r_{u}} \frac{d y}{r_{u}}+\frac{d}{d_{x}} \varrho \alpha_{m} U_{s m}^{2} r_{\alpha}^{2} \int_{0}^{1}\left(\frac{U_{s}}{U_{s m}}\right)^{2} \frac{\alpha}{\alpha_{m}} \frac{y}{r_{\alpha}} \frac{d y}{r_{\alpha}}-
$$




$$
\begin{aligned}
& -U_{0,5} \frac{d}{d x} \varrho U_{m} r_{\alpha}^{2} \int_{0}^{1} \frac{U}{U_{m}} \frac{y}{r_{\alpha}} \frac{d y}{r_{\alpha}}-U_{s 0,5} \frac{d}{d x} \varrho \alpha_{m} U_{s m} r_{\alpha}^{2} \times \\
& \times \int_{0}^{1} \frac{\alpha}{\alpha_{m}} \frac{U_{s}}{U_{s m}} \frac{y}{r_{\alpha}} \frac{d y}{r_{\alpha}}=\left.k \varrho U_{m}^{2} r_{\alpha} \frac{1+0,5 n^{2} \alpha_{m}}{1+0,5 \alpha_{m}} \frac{d U / U_{m}}{d y / r_{u}}\right|_{0,5} .
\end{aligned}
$$

Аналогично приводятся к безразмерному виду уравнения (2):

$$
\begin{gathered}
\frac{d}{d x} \varrho U_{m}^{2} r_{u}^{2} \int_{0}^{\infty}\left(\frac{U}{U_{m}}\right)^{2} \frac{y}{r_{u}} \frac{d y}{r_{u}}+\frac{d}{d x} \varrho \alpha_{m} U_{s m}^{2} r_{\alpha}^{2} \int_{0}^{\infty}\left(\frac{U_{s}}{U_{s m}}\right)^{2} \frac{\alpha}{\alpha_{m}} \frac{y}{r_{\alpha}} \frac{d y}{r_{\alpha}}=0 \\
\frac{d}{d x} \varrho U_{s m}^{2} \alpha_{m} r_{\alpha}^{2} \int_{0}^{\infty}\left(\frac{U_{s}}{U_{s m}}\right)^{2} \frac{\alpha}{\alpha_{m}} \cdot \frac{y}{r_{\alpha}} \frac{d y}{r_{\alpha}}=18 L \varrho \frac{v}{\delta^{2}} \frac{\varrho}{\varrho_{s}} \alpha_{m} \times \\
\times\left(U_{m} r_{\alpha}^{2} \int_{0}^{\infty} \frac{U}{U_{m}} \frac{\alpha}{\alpha_{m}} \frac{y}{r_{\alpha}} \frac{d y}{r_{\alpha}}-U_{s m} r_{\alpha}^{2} \int_{0}^{\infty} \frac{U_{s}}{U_{s m}} \frac{\alpha}{\alpha_{m}} \frac{y}{r_{\alpha}} \frac{d y}{r_{\alpha}}\right) \\
\frac{d}{d x} \varrho \alpha_{m} U_{s m} r_{\alpha}^{2} \int_{0}^{\infty} \frac{\alpha}{\alpha_{m}} \frac{U_{s}}{U_{s m}} \frac{y}{r_{\alpha}} \frac{d y}{r_{\alpha}}=0
\end{gathered}
$$

Введем обозначения

$$
\begin{array}{ll}
\int_{0}^{\infty} \frac{\alpha}{\alpha_{m}} \frac{U_{s}}{U_{s m}} \frac{y}{r_{\alpha}} \frac{d y}{r_{\alpha}}=A ; \quad \int_{0}^{\infty}\left(\frac{U}{U_{m}}\right)^{2} \frac{y}{r_{u}} \frac{d y}{r_{u}}=B ; \\
\int_{0}^{\infty}\left(\frac{U_{s}}{U_{s m}}\right)^{2} \frac{\alpha}{\alpha_{m}} \frac{y}{r_{\alpha}} \frac{d y}{r_{\alpha}}=B_{s} ; & \int_{0}^{1} \frac{U}{U_{m}} \frac{y}{r_{\alpha}} \frac{d y}{r_{\alpha}}=A_{1} ; \\
\int_{0}^{r_{\alpha} r_{u}}\left(\frac{U}{U_{m}}\right)^{2} \frac{y}{r_{u}} \frac{d y}{r_{u}}=B_{1} ; & \int_{0}^{1}\left(\frac{U_{s}}{U_{s m}}\right)^{2} \frac{\alpha}{\alpha_{m}} \frac{y}{r_{\alpha}} \frac{d y}{r_{\alpha}}=B_{s 1} ; \\
\int_{0}^{\infty} \frac{\alpha}{\alpha_{m}} \frac{U}{U_{m}} \frac{y}{r_{\alpha}} \frac{d y}{r_{\alpha}}=F . &
\end{array}
$$

Особенность интегральных методов заключается в том, что необходимые для определения $A_{i}, B_{i}$ и $F$ функции распределения параметров в поперечном сечении задаются заранее чаще на основании опытных данных, иногда в результате интегрирования дифференциальных уравнений для течений, близких по типу к исследуемым. Такой подход оправдан потому, что для автомодельных течений, к каковым на достаточном удалении от сопла относится струя, интегралы $A_{i}$ и $B_{i}$, как правило, являются константами, значения которых мало зависят от вида функции распределения.

Зададим функции раопределения в виде 


$$
\frac{D}{D_{m}}=\exp \left[-0,7\left(\frac{r}{r_{0,5 D}}\right)^{n_{D}}\right]
$$

Опыты показали, что на основном участке струи с точностью, достаточной для практических расчетов, $n_{u}=1,75, n_{\alpha}=1,33, n_{s}=2$. Отсюда

$$
\begin{gathered}
A=\int_{0}^{\infty} \exp \left[-0,7\left(\frac{r}{r_{\alpha}}\right)^{1,33}-0,7\left(\frac{r}{r_{\alpha}}\right)^{2}\left(\frac{r_{\alpha}}{r_{u s}}\right)^{2}\right] \frac{y}{r_{\alpha}} \frac{d y}{r_{\alpha}}=0,83, \\
B=\int_{0}^{\infty} \exp \left[-1,4\left(\frac{r}{r_{u}}\right)^{1,75}\right] \frac{y}{r_{u}} \cdot \frac{d y}{r_{u}}=0,368 \\
A_{1}=\int_{0}^{1} \exp \left[-0,7\left(\frac{r}{r_{u}}\right)^{1,75}\left(\frac{r_{u}}{r_{\alpha}}\right)^{1,75}\right] \frac{y}{r_{\alpha}} \cdot \frac{d y}{r_{\alpha}}=0,0715, \\
B_{s}=\int_{0}^{\infty} \exp \left[-0,7\left(\frac{r}{r_{\alpha}}\right)^{1,33}-1,4\left(\frac{r}{r_{\alpha}}\right)^{2}\left(\frac{r_{\alpha}}{r_{u s}}\right)^{2}\right] \frac{y}{r_{\alpha}} \frac{d y}{r_{\alpha}}=0,661, \\
B_{s 1}=\int_{0}^{1} \exp \left[-0,7\left(\frac{r}{r_{\alpha}}\right)^{1,33}-1,4\left(\frac{r}{r_{\alpha}}\right)^{2}\left(\frac{r_{\alpha}}{r_{u s}}\right)^{2}\right] \frac{y}{r_{\alpha}} \frac{d y}{r_{\alpha}}=0,296, \\
F=\int_{0}^{\infty} \exp \left[-0,7\left(\frac{r}{r_{\alpha}}\right)^{1,33}-0,7\left(\frac{r}{r_{u}}\right)^{1,75}\left(\frac{r_{u}}{r_{\alpha}}\right)^{1,75}\right] \frac{y}{r_{\alpha}} \frac{d y}{r_{u}}=0,0667,
\end{gathered}
$$

После подстановки соотношений (9) в уравнения (5) и (6) и вычислений имеем окончательно

$$
\begin{gathered}
\frac{d}{d x} \varrho \alpha_{m} U_{s m} r_{u}^{2}=0, \\
\frac{d}{d x} \alpha_{m} U_{s m}^{2} r_{u}^{2}+3,48 \frac{d}{d x} U_{m}^{2} r_{u}^{2}=0, \\
\frac{d}{d x} \alpha_{m} U_{s m}^{2} r_{u}^{2}=\frac{3,1 \cdot 10^{-2} v}{\delta^{2}} L r_{u}^{2} \alpha_{m}\left[U_{m}-1,02 U_{s m}\right], \\
\frac{d}{d x} \alpha_{m} U_{s m}^{2} r_{u}^{2}+1,405 \frac{d}{d x} r_{u}^{2} U_{m}^{2}-1,31 U_{m} \frac{d}{d x} U_{m} r_{u}^{2}= \\
=-0,104 r_{u} U_{m}^{2} \frac{1+n^{2} 0,5 \alpha_{m}}{1+0,5 \alpha_{m}} .
\end{gathered}
$$

На рисунке сопоставлены результаты расчета по уравнениям (10) с экспериментом. Кривые совмещались в сечении $x=350$ мм $\left(x / d_{0} \simeq\right.$ $\simeq 11)$, где по данным эксперимента начинается подобие. Удовлетворительное совпадение получено при $k=0,022$ и силе сопротивления в два раза большей, чем следует из формулы (3). Несколько меньщие значе- 


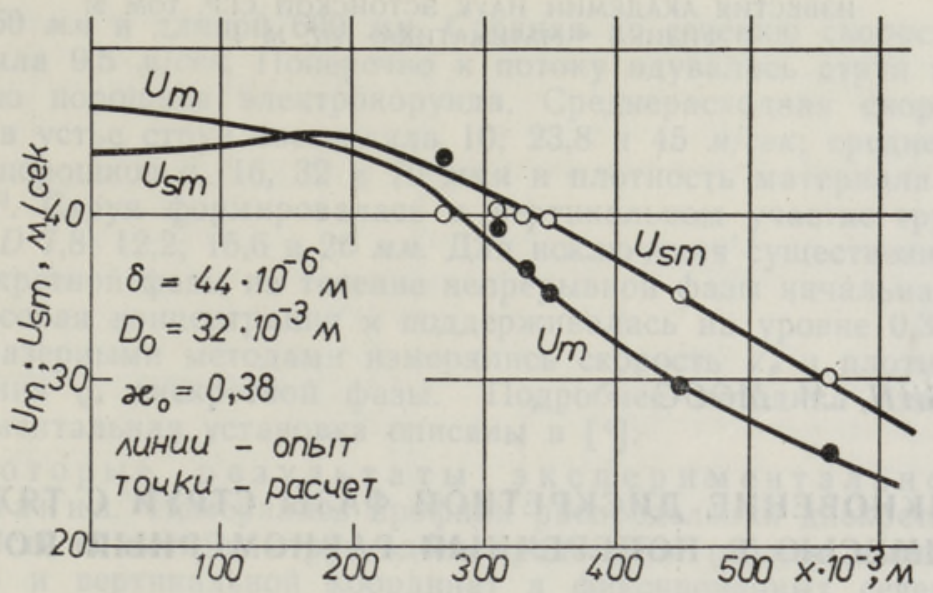

ния $k$ для струй с трубным профилем, по сравнению со струями с равномерным начальным профилем, общеизвестны и получены при исследовании однофазных струй. Существенное увеличение коэффициента сопротивления частиц можно объяснить их неправильной формой и вращением. Расчеты по формулам (10) требуют на машине «Наири» в 10 раз меньше времени, чем по формулам $\left[{ }^{4}\right]$.

\section{Л ИТЕРАТ У Р А}

1. Патанкар С., Сполдинг Д., Тепло- и массообмен в пограничных слоях, M., 1971 , с. 127.

2. Гр одзо вски й Г. Л., Уч. зап. ЦАГИ, V, № 2, 80 (1974).

3. Абрамович Г. Н., Бажанов В. И., Гиршович Т. А., Изв. АН СССР, МЖГ, № 5, 41 (1972).

4. Л а а т с М. К., Р оз енштей А. 3., Фриш ман Ф. А., В кн.: Турбулентные двухфазные течения, Таллин, 1976.

5. Гин е в ски й А. С., Теория турбулентных струй и следов, М., 1969, с. 400.

6. А б р а м ов и ч Г. Н., Докл. АН СССР, 190, № 5, 1052 (1970).
Ннститут термофизики и электрофизики
Академии наук Эстонской ССР
Поступила в редакцию 3/XI 1976

V. VANATOA, M. LAATS, A. KARTUSINSKI, F. FRISMAN

\section{TASAKAALUSTAMATU VABA HIIBSE VOOLUSE ARVUTAMISE INTEGRAALMEETOD}

Esitatakse mehaaniliselt tasakaalustamatu vaba hiibse (gaas - tahked osakesed) vooluse arvutus. Seejuures on kasutatud iselaadset integraalsete seoste meetodit, kus summaarse liikumishulga vōrrandid kehtivad ka mingi vooluse osa jaoks. Arvutuse tulemused on kõrvutatud katsetulemustega.

\section{VANATOA, M. LAATS, A. KARTUSHINSKI, F. FRISHMAN}

\section{AN INTEGRAL METHOD FOR CALCULATING AN AXISYMMETRICAL TWO-PHASE JET}

Solution of the problem of the developed zone of nonequilibrious axisymmetric turbulent jet containing fine solid admixture is presented. Integral equations of mass of admixture, momentum of solid phase and summary momentum are used. The latter is presented not only for the whole space but also for the line of half-level of the concentration. Results of calculations are compared with experimental results of other authors. 